\title{
Ocorrência de nematóides fitoparasitos em plantas ornamentais nos Estados de São Paulo e Minas Gerais ${ }^{(1)}$
}

\author{
CLAUDIO MARCELO G. OLIVEIRA(2); ROBERTO K. KUBO(2); SONIA R. ANTEDOMENICO(2); \\ AILTON R. MONTEIRO ${ }^{(3)}$ e MARIO M. INOMOTO( ${ }^{(3)}$
}

\begin{abstract}
Resumo
O objetivo do presente trabalho foi o de discutir a importância das principais espécies de nematóides encontradas associadas a plantas ornamentais cultivadas nos Estados de São Paulo e Minas Gerais, com base em dados de distribuição geográfica, de novas ocorrências e relativos à sintomatologia. Nematóides foram extraídos de $5 \mathrm{~g}$ de raízes e identificados sob microscópio. As espécies mais freqüentes foram Meloidogyne javanica (38\% das amostras), $M$. incognita (19\%) e Helicotylenchus dihystera (11\%). Constituíram novas ocorrências no Brasil: M. incognita em Gloxinia sp., Arundina graminifolia (orquídea-bambu) e Aptenia cordifolia (rosinha do sol); $M$. javanica em A. graminifolia, Hibiscus spp., Gloxinia sp., Eustoma grandiflorum (lisianto), Heliconia rostrata (falsa ave do paraíso), Graptophyllum pictum (graptofilo), Holmskioldia sanguinea (chapéu-chinês) e Exacum affine (violeta-alemã); Pratylenchus brachyurus em E. grandiflorum, Cattleya sp. (orquídea) e diferentes cultivares de Lilium sp. (lírio); Helicotylenchus dihystera em E. grandiflorum, Lilium sp., Impatiens balsamina e A. cordifolia; H. multicinctus em Pachystachys lutea (camarão amarelo); H. pseudorobustus em Heliconia sp., e Cactodera cacti em Schlumbergera sp. (flor de maio).
\end{abstract}

Palavras-chaves: Brasil, levantamento, nematóide das galhas, nematóide das lesões, nematóides espiralados, plantas ornamentais.

\section{Abstract \\ Occurrence of plant parasitic nematodes on ornamental plants in São Paulo and Minas Gerais States, Brazil}

\begin{abstract}
The objective of this work was to report the geographic distribution, new occurrences and symptoms caused by the main species of nematodes collected from ornamental plants cultivated in the States of São Paulo and Minas Gerais, Brazil. Nematodes were extracted from $5 \mathrm{~g}$ of roots by centrifugation method and identified under microscope with the aid of a camera lucida for measurements. The most frequently occurring species were Meloidogyne javanica (38\% of all samples), M. incognita (19\%) and Helicotylenchus dihystera (11\%). Among the identified species, the following associations constitute new records to Brazil: M. incognita in Gloxinia sp., Arundina graminifolia and Aptenia cordifolia; M. javanica in A. graminifolia, Hibiscus spp., Gloxinia sp., Eustoma grandiflorum, Heliconia rostrata, Graptophyllum pictum, Holmskioldia sanguinea and Exacum affine; Pratylenchus brachyurus in E. grandiflorum, Cattleya sp. and different cultivars of Lilium sp.; Helicotylenchus dihystera in E. grandiflorum, Lilium sp., Impatiens balsamina and A. cordifolia; H. multicinctus in Pachystachys lutea; H. pseudorobustus in Heliconia sp. and Cactodera cacti in Schlumbergera sp.
\end{abstract}

Key words: first record, lesion nematode, ornamental plants, root-knot nematode, spiral nematode, survey

\section{INTRODUÇÃO}

No Brasil, o mercado de flores e plantas ornamentais encontra-se em expansão, movimentando em torno de 800 milhões de dólares/ano e estimulando produtores a investir nessa área (JUNQUEIRA e PEETZ, 2005). São Paulo é o principal Estado produtor respondendo por aproximadamente $70 \%$ da produção nacional, seguido de Santa Catarina, Rio Grande do Sul e Minas Gerais
(IBRAFLOR, 2001; JUNQUEIRA e PEETZ, 2002). No entanto, há fatores relacionados aos aspectos sanitários que limitam o seu cultivo, com destaque ao ataque, por vezes severo, de nematóides.

Os nematóides associados às plantas ornamentais parasitam principalmente os órgãos subterrâneos (raízes, rizomas, tubérculos e bulbos), mas também a parte aérea (caules e folhas). As perdas causadas são de dois tipos: redução de produção e depreciação da qualidade do produto a ser comercializado.

\footnotetext{
(1) Recebido para publicação em 20/12/2006 e aceito em 27/03/2007.

(2) Centro Experimental Central Instituto Biológico, Caixa Postal 70, 13001-970, Campinas (SP), Brasil.

(3) Departamento de Entomologia, Fitopatologia e Zoologia Agrícola, Escola Superior de Agricultura "Luiz de Queiroz", Universidade de São Paulo, Caixa Postal 9, 13418-900, Piracicaba (SP), Brasil.
} 
No Brasil, mais de 20 gêneros de fitonematóides foram detectados em associação a plantas ornamentais (SILVEIRA et al., 1986; COSTA MANSO et al., 1994; SILVEIRA, 1997; WILCKEN e FERRAZ, 1998; COSTA et al., 2001; OLIVEIRA, 2001; LINS e COELHO, 2004; OLIVEIRA, 2006; OLIVEIRA e KUBO, 2006). De forma geral, as espécies pertencentes aos gêneros Meloidogyne, Pratylenchus, Radopholus e Aphelenchoides são consideradas as principais no Brasil devido à freqüência com que ocorrem e à intensidade das perdas causadas (OLIVEIRA, 2001; 2006). Porém, o conhecimento disponível na literatura sobre a ocorrência de nematóides em plantas ornamentais é ainda limitado. Afora relatos isolados de novas ocorrências, a literatura brasileira registra apenas três levantamentos de nematóides em plantas ornamentais, um em Lavras, MG (COSTA et al., 2001), outro no norte e noroeste do Paraná (MORITA et al., 2006) e outro na zona da mata de Pernambuco (LINS e COELHO, 2004). Portanto, ainda não há referência de nematóides associados a um grande número de espécies de plantas ornamentais nas diferentes regiões geográficas brasileiras.

Assim, o presente trabalho foi elaborado com o objetivo de discutir a importância das principais espécies de nematóides pertencentes aos gêneros Cactodera, Helicotylenchus, Meloidogyne e Pratylenchus encontradas em raízes de plantas ornamentais cultivadas nos Estados de São Paulo e Minas Gerais, com base em dados de distribuição geográfica, de novas ocorrências e relativos à sintomatologia.

\section{MATERIALEMÉTODOS}

O presente inclui resultados de coletas nematológicas realizadas durante visitas técnicas a propriedades localizadas nos municípios paulistas de Artur Nogueira, Campinas, Guarujá, Holambra, Paranapanema, Piracicaba, Registro e Timburi, além de Andradas e Munhoz estes do Estado de Minas Gerais, em condições de campo, estufas ou jardins. Cada amostra foi formada por raízes e solo da rizosfera, coletados à profundidade de 0 a $20 \mathrm{~cm}$, com auxílio de enxadão, pá de jardim e tesoura de poda. As amostras foram acondicionadas em sacos de polietileno, etiquetadas e guardadas em caixas térmicas para transporte ao laboratório.

Também estão registradas, no presente trabalho, as amostras encaminhadas para análise nematológica às clínicas mantidas pelos laboratórios de Nematologia do Departamento de Entomologia, Fitopatologia e Zoologia Agrícola da Escola Superior de Agricultura "Luiz de Queiroz" (ESALQ/USP), em Piracicaba (SP), e do Centro Experimental Central do Instituto Biológico (CEIB/Instituto Biológico), em Campinas (SP), no período de 1997 a 2006.

Os espécimes de nematóides foram extraídos de $5 \mathrm{~g}$ de raízes pelo método de COOLEN e D'HERDE(1972). O volume de suspensão obtido foi reduzido, por sifonamento, a $10 \mathrm{~cm}^{3}$ e uma alíquota de $1 \mathrm{~cm}^{3}$ foi utilizada para a contagem dos nematóides presentes, em lâmina de Peters, sob microscópio óptico.
Para a identificação das espécies, usaram-se lâminas temporárias e/ou permanentes examinadas em microscópio. A identificação de espécies de Meloidogyne foi realizada com a análise da configuração perineal de fêmeas adultas (HARTMAN e SASSER, 1985). As espécies de Pratylenchus foram identificadas com base em HANDOO e GOLDEN (1989); LOOF (1991); ROMÁN e HIRSCHMANN (1969) e as de Cactodera e Helicotylenchus com as chaves de GRANEY e BIRD (1990) e SHER (1966), respectivamente.

\section{RESULTADOS E DISCUSSÃO}

Nas amostras analisadas, encontraram-se nematóides das galhas (Meloidogyne arenaria, M. hapla, $M$. javanica e $M$. incognita), das lesões radiculares (Pratylenchus brachyurus e $P$. zeae), espiralados (Helicotylenchus dihystera, H. multicinctus e $H$. pseudorobustus) e o nematóide-de-cisto do cactus (Cactodera cacti). Destacaram-se as seguintes novas ocorrências no Brasil: M. incognita em Gloxinia sp., Arundina graminifolia e Aptenia cordifolia; $M$. javanica em A. graminifolia, Hibiscus spp., Gloxinia sp., Eustoma grandiflorum, Heliconia rostrata, Graptophyllum pictum, Holmskioldia sanguinea e Exacum affine; Pratylenchus brachyurus em E. grandiflorum, Cattleya sp. e diversas cultivares de Lilium sp.; P. zeae em Rosa sp.; Helicotylenchus dihystera em E. grandiflorum, Lilium sp., Impatiens sp. e A. cordifolia; H. multicinctus em Pachystachys lutea; H. pseudorobustus em Heliconia sp.; e Cactodera cacti em Schlumbergera sp. (tabela 1).

\subsection{CONSIDERAÇÕES SOBRE OS NEMATÓIDES DAS GALHAS - MELOIDOGYNE SPP.}

Meloidogyne javanica foi a mais freqüente, presente em 38\% das amostras analisadas (tabela 1). Atingiu maiores densidades populacionais nas raízes de lisianto (Eustoma grandiflorum), cravo (Dianthus caryophilus), Impatiens balsamina e Exacum affine (tabela 1). No geral, as plantas mostravam galhas no sistema radicular e desenvolvimento insatisfatório (figura 1). Em alguns casos mais graves, como ataque de $M$. javanica a cravo em estufa, observaram-se reboleiras formadas por plantas de porte reduzido, comprometendo a produção e comercialização das flores de corte (figura 2).

Os sintomas em plantas de lisianto provenientes de Artur Nogueira e Ibiúna, SP (figura 1A) evidenciam o potencial de perdas por $M$. javanica nessa ornamental. Recentemente, na Califórnia, EUA, sob condições controladas, demonstraram-se os efeitos adversos decorrentes de ataques por diferentes densidades populacionais de Meloidogyne hapla, $M$. incognita e $M$. javanica sobre plantas de lisianto cv. Mariachi Lime Green. $M$. javanica foi a espécie que mais se multiplicou, seguida de M. incognita. Também, por ocasião da colheita, observou-se que o número médio de flores produzidas foi reduzido em até $58 \%$, quando comparadas plantas cultivadas com e sem a presença dos nematóides 
(SCHOCHOW et al., 2004).

No presente trabalho, diagnosticou-se $M$. javanica (1050-1340 ovos + juvenis/g de raiz) e $M$. incognita (60-2110 ovos + juvenis/g de raiz) em Impatiens balsamina cultivada em jardins no município de Campinas (SP). Anteriormente, esses nematóides haviam sido relatados nessa ornamental no estado do Ceará (PONTE, 1968) e no Distrito Federal (PONTE et al., 1975). De fato, o nematóide das galhas é freqüentemente encontrado causando danos a Impatiens balsamina em diversas regiões tropicais e subtropicais. Por exemplo, na Venezuela, MONTILLA et al. (2000) avaliaram as reações de três cultivares (Dazzler Blush, Dazzler Orange e Showstopper Cherry), 45 dias após o substrato ser infestado com 10 ovos de $M$. incognita por $\mathrm{cm}^{3}$. Independentemente das cultivares, os autores observaram diferenças significativas na massa fresca das raízes infectadas em relação às respectivas testemunhas não infectadas, com reduções de até $65 \%$. Clorose generalizada foi observada nas plantas, embora a massa da parte aérea não tenha sido afetada.

Meloidogyne hapla é considerada a mais importante do gênero associada à roseira, sendo tal associação relatada nas principais áreas produtoras do mundo. No presente estudo, M. hapla foi encontrada parasitando raízes de roseira em Munhoz e Andradas, MG (figura 1B). No Brasil, FREIRE e PONTE (1976) assinalaram Meloidogyne sp. em associação à roseira cvs. Queen Elisabeth e Tifani, no estado da Bahia, enquanto que SILVEIRA et al. (1986) encontraram M. hapla, M. javanica, M. arenaria e Meloidogyne sp. em roseiras provenientes de Jaguariúna (SP).

\subsection{CONSIDERAÇÕES SOBRE OS NEMATÓIDES DAS LESÕES RADICULARES - PRATYLENCHUS SPP.}

Embora espécie polífaga e cosmopolita, não havia registro de $P$. brachyurus parasitando raízes de orquídea (Cattleya sp.), lisianto, hibisco e diferentes cultivares de lírio (tabela 1). Em material vegetal composto de dez plantas de Cattleya sp., proveniente de Paranapanema, SP, verificou-se a presença de $P$. brachyurus nas raízes. Anteriormente, $P$. brachyurus foi relatado associado a outra orquidácea, Vanilla fragrans, em Tonga, na Polinésia, causando a redução do crescimento (BRIDGE, 1988). Futuros experimentos deverão ser realizados para comprovar a patogenicidade e os danos de P. brachyurus às plantas ornamentais.

Embora sem causar perdas aparentes, detectouse também pela primeira vez $P$. zeae em raízes de roseiras provenientes de Holambra, SP. Nessa cultura, uma outra espécie, $P$. vulnus, é particularmente importante, ocasionando declínio das plantas em muitas regiões do mundo (LEHMAN, 1982). No Brasil, ocorre em roseirais de Minas Gerais e São Paulo, mas não se conhece a sua real distribuição nesses Estados. ROSSI et al. (2000) assinalaram $P$. vulnus em roseiras cv. Verônica enxertadas sobre Rosa multiflora, cultivadas em casas de vegetação, em Itapeva, MG. As plantas tinham porte reduzido, clorose e não respondiam à adubação de maneira satisfatória.

\subsection{CONSIDERAÇÕES SOBRE OS NEMATÓIDES ESPIRALADOS-HELICOTYLENCHUS SPP.}

Helicotylenchus multicinctus foi encontrado parasitando raízes de camarão-amarelo cultivado em jardim residencial no município do Guarujá, SP e $H$. pseudorobustus foi detectado em associação a Heliconia sp. (vermelha) proveniente de Registro (SP). Anteriormente, ZEM e LORDELLO (1983) haviam relatado $H$. multicinctus em Heliconia sp. e Musa ornata, enquanto que LINS e COELHO (2004) verificaram a ocorrência de doenças em plantas ornamentais tropicais no Estado de Pernambuco e encontraram Helicotylenchus sp. em várias ornamentais pertencentes às famílias Musaceae, Heliconeaceae e Zinziberaceae.

No presente trabalho, detectou-se $H$. dihystera associado a lisianto, lírio, Impatiens e rosinha do sol (figura 3). Essa espécie ectoparasita apresenta ampla gama de plantas hospedeiras (COSTA MANSO et al., 1994), incluindo ornamentais. Por exemplo, COSTA et al. (2001) analisaram solo e raízes de 56 plantas ornamentais cultivadas em Lavras (MG), e H. dihystera ocorreu em 15,63\% das amostras.

\subsection{CONSIDERAÇÕESSOBRE ONEMATÓIDE-DE- CISTO DO CACTUS - CACTODERA CACTI}

Cistos, fêmeas e juvenis de Cactodera cacti foram encontrados em plantas de cactus provenientes de Jundiaí e Jacareí, SP (tabela 1). De forma semelhante, SANTOS et al. (2001) relataram a ocorrência de Heterodera cacti $(=C$. cacti $)$ em alguns lotes de cactáceas ornamentais cultivadas na região de Jundiaí, SP. Essa espécie de nematóide formador de cisto tem ampla distribuição geográfica mundial, ocorrendo em praticamente todas as áreas em que plantas de cactus são cultivadas, causando perdas relevantes. Por exemplo, em flor-de-maio (Schlumbergera sp.), causa desenvolvimento insatisfatório das plantas, devido à diminuição do número de flores e pela coloração avermelhada da parte aérea (RICHARDSON e GRAWAL, 1993).

\section{CONCLUSÃO}

O controle de nematóides parasitos de plantas ornamentais é provavelmente uma das atividades que mais desafiam os produtores. Por exemplo, com exceção do uso de fenamifós que pode ser utilizado no tratamento de marantas infestadas com Radopholus similis (BARBOSA et al., 1990), não há registros de nematicidas recomendados para plantas ornamentais no Brasil, restringindo a sua prática. Como alternativa, o controle biológico apresentase com grande potencial de uso. SOARES e SANTOS (2005) recomendam o uso de um coquetel de cinco fungos (Arthrobotrys oligospora, A. musiformis, Dactylella 
leptospora, Monacrosporium robustum e Paecilomyces lilacinus) aplicado no substrato para produção de mudas de crisântemo. Segundo os autores, mudas de 20 dias de idade inoculadas com fungos e mudas sem tratamento foram transplantadas em estufa infestada por Meloidogyne javanica. Os canteiros implantados com mudas produzidas em substrato tratado exibiram crescimento e florescimento uniforme de plantas, enquanto que canteiros implantados com mudas convencionais sofreram severas perdas.

De forma geral, devido às limitações do controle curativo, as medidas preventivas são preconizadas, incluindo-se entre elas, o uso de mudas isentas de nematóides e plantio em solo ou substrato não infestado.

\section{REFERÊNCIAS}

BARBOSA, L. J.; BENATTI, A. M.; SALVO, S. Estudo da eficiência de nematicida para o controle de Radopholus similis (Cobb, 1893) na cultura da maranta, Calathea zebrina (Maranta zebrina). Ecossistema, Espírito Santo do Pinhal, v.15, p.160-66, 1990.

BRIDGE, J. Plant-parasitic nematode problems in the Pacific Islands. Journal of Nematology, Lawrence, v.20, n.2, p.17383, 1988.

COOLEN, W. A.; J. D'HERDE, C. J. A method for the quantitative extraction of nematodes from plant tissue. Ghent: State Nematology and Entomology Research Station, p.77, 1972.

COSTAMANSO, E.; TENENTE, R. C. V.; FERRAZ, L. C. C. B., OLIVEIRA, R. S.; MESQUITA, R. Catálogo de nematóides fitoparasitos encontrados associados a diferentes tipos de plantas no Brasil. Embrapa, Centro Nacional de Pesquisa de Recursos Genéticos e Biotecnologia. Brasília: Embrapa-SPI, 1994. 488p.

COSTA, M. J. N.; OLIVEIRA, S.; COELHO, S. J., CAMPOS, V. P. Nematóides em plantas ornamentais. Ciência e Agrotecnologia, Lavras, v.25, p.1127-32, 2001.

FREIRE, F. C. O.; PONTE, J. J. Nematóides das galhas, Meloidogyne spp., associados ao parasitismo de plantas no Estado da Bahia. Boletim Cearense de Agronomia, Fortaleza, v.17, p.47-55, 1976.

GRANEY, L. S. O.; G. W. BIRD. Descriptions and comparative morphology of Cactodera milleri, new species, (Nematoda: Heteroderidae) and Cactodera cacti with a review and key to the genus Cactodera. Journal of Nematology, Lawrence, v.22, n.4, p.457-80, 1990.

HANDOO, Z. A., GOLDEN, M. A. A key and diagnostic compendium to the species of the genus Pratylenchus Filipjev. Journal of Nematology, Lawrence, v.21, p.202-18, 1989.

HARTMAN, K. M.; SASSER, J. N. Identification of Meloidogyne species on the basis of diferential host test and perineal-pattern morphology. In: BARKER, K. R.; CARTER, C. C.; SASSER, J. N. (Eds.) An advanced treatise on Meloidogyne. II. Methodology. Raleigh: Department of Plant Pathology, North Carolina State University, 1985. p.69-77.

IBRAFLOR, Brasil: mostra tua flora. Informativo, v.7, n.23, p.4, 2001. Disponível em: <http://www.uesb.br/flower/ IBRAFLOR.PDF>. Acesso em: 22 nov. 2006.

JUNQUEIRA, A. H.; PEETZ, M. S. Os pólos de produção de flores e plantas ornamentais do Brasil: uma análise do potencial exportador. Revista Brasileira de Horticultura Ornamental, Campinas, v.8, n.1-2, p.25-47, 2002.

JUNQUEIRA, A. H.; PEETZ, M. S. Mercado brasileiro de flores e plantas ornamentais - perspectivas e tendências. Informativo Ibraflor, Holambra, v.11, n.45, p.8, 2005.

LEHMAN, P. S. Diseases of roses caused by nematodes. Gainesville: Florida Department of Agriculture \& Consumer.Services, p.2, 1982.

LINS, S. R. O.; COELHO, R. S. B. Ocorrência de doenças em plantas ornamentais tropicais no estado de Pernambuco. Fitopatologia Brasileira, Brasília, v.29, n.3, p.332-35, 2004.

LOOF, P. A. A. The family Pratylenchidae Thorne, 1949. In: NICKLE, W. R. (Ed.) Manual of agricultural nematology. New York: Marcel Dekker, 1991. p.363-421.

MONTILLA, J.; RENAUD, J; JIMENEZ, N, BRICENO; E. Susceptibility of Impatiens balsamina cultivars to rootknot nematode, Meloidogyne incognita. Fitopatologia Venezolana, Maracay, v.13, n.2, p. 41-42, 2000.

MORITA, D. A. S.; DIAS-ARIEIRA, C. R.; MOLINA, S. C.; OBICI, L. V.; SANTOS, L. L.; MACHADO, M. H. Levantamento de nematóides em plantas ornamentais no Norte e Noroeste do Paraná. Fitopatologia Brasileira, Brasília, v.31, suplemento, p.179, 2006.

OLIVEIRA, C. M. G. Nematóides parasitos de plantas. In: PRAGAS EDOENÇAS EM PLANTAS ORNAMENTAIS, IMENES, S. L.; ALEXANDRE, M. A. V. (Eds) CD-ROM (ISBN 85-88694-01-8). Instituto Biológico, São Paulo, p.3847,2001

OLIVEIRA, C. M. G.; KUBO, R. K. Novos assinalamentos de nematóides de parte aérea (Aphelenchoides spp.) em plantas de begônia no Brasil. Revista Brasileira de Horticultura Ornamental, Campinas, v.12, p.134-137, 2006.

OLIVEIRA, C. M. G. Nematóides Parasitos de Plantas Ornamentais no Brasil. Fitopatologia Brasileira, Lavras, v.31:S117-S118, 2006.

PONTE, J. J. Subsídios ao conhecimento das plantas hospedeiras e ao controle dos nematóides das galhas, Meloidogyne spp., no Estado do Ceará. Boletim Cearense de Agronomia, Fortaleza, v.9, p.1-26, 1968.

PONTE, J. J.; MATTOS, J. K. A.; TENENTE, R. C. V., MARIA, L. Primeira lista de hospedeiros de Meloidogyne do Distrito Federal. (Brasil). Revista Sociedade Brasileira Fitopatologia, Mossoró, v. 6/8, p. 29-34, 1975.

RICHARDSON, P. N.; GREWAL, P. S. Nematode pests of glasshouse crops and mushrooms. In: Evans, K., Trudgill, D.L. \& Webster, J.M. (eds). Plant parasitic nematodes in temperate agriculture. Wallinford: CAB International, 1993. p.501-44. 
ROMÁN, B., HIRSCHMANN, H. Morphology and morphometrics of six species of Pratylenchus. Journal of Nematology, Lawrence, v.1, p.363-86, 1969.

ROSSI, C. E.; CALDARI JR., P.; MONTEIRO, A. R. Ocurrence of Pratylenchus vulnus on rose in Minas Gerais State, Brazil. Arquivos do Instituto Biológico, São Paulo, v.67, n.1, p.147-48, 2000.

SANTOS, J. M.; PREZOTTO, T.; MAIA, A. S. Detecção de Heterodera cacti em São Paulo. Nematologia Brasileira, Piracicaba, v.25, p.139-40, 2001.

SCHOCHOW, M.; TJOSVOLD, S. A.; PLOEG, A. T. Host status of Lisianto 'Mariachi Lime Green' for three species of root-knot nematodes. HortScience, Alexandria, v.39, p.120-23, 2004.

SHER, S. A. Revision of the Hoplolaiminae (Nematoda) VI. Helicotylenchus (Steiner, 1945). Nematologica, Leiden, v.12, n. , p.1-56, 1966.

SILVEIRA, S. G. P.; CURI, S. M.; STEFANINI, P. C. Nematóides de plantas detectados pela Seção de Nematologia do Instituto Biológico de São Paulo, Brasil.
Biológico, São Paulo, v.52, p.91-104, 1986.

SILVEIRA, S. G. P. Nematóides em plantas ornamentais no Estado de São Paulo. In: ALMEIDA, I. M. G., MALAVOLTA JÚNIOR, V. A., IMENES, S. L. (Eds). Problemas fitossanitários em plantas ornamentais. Campinas, Instituto Biológico, 1997. p.33-39.

SOARES, P. L. M., SANTOS, J. M. Controle biológico de Meloidogyne javanica em crisântemo de corte em ambiente protegido. Horticultura Brasileira, Brasília, v. 23, n. 2, suplemento, p. 531, 2005.

WILCKEN, S. R. S., FERRAZ, L. C. C. B. Reprodução de espécies de Meloidogyne e Pratylenchus (Nemata: Tylenchoidea) em diferentes tipos de plantas ornamentais. Summa Phytopathologica, Jaboticabal, v.24, p.171-76, 1998. ZEM,A. C., LORDELLO, L. G. E. Estudos sobre hospedeiros de Radopholus similis e Helicotylenchus multicinctus. Sociedade Brasileira de Nematologia, Piracicaba, v. 7, p.175-88, 1983.

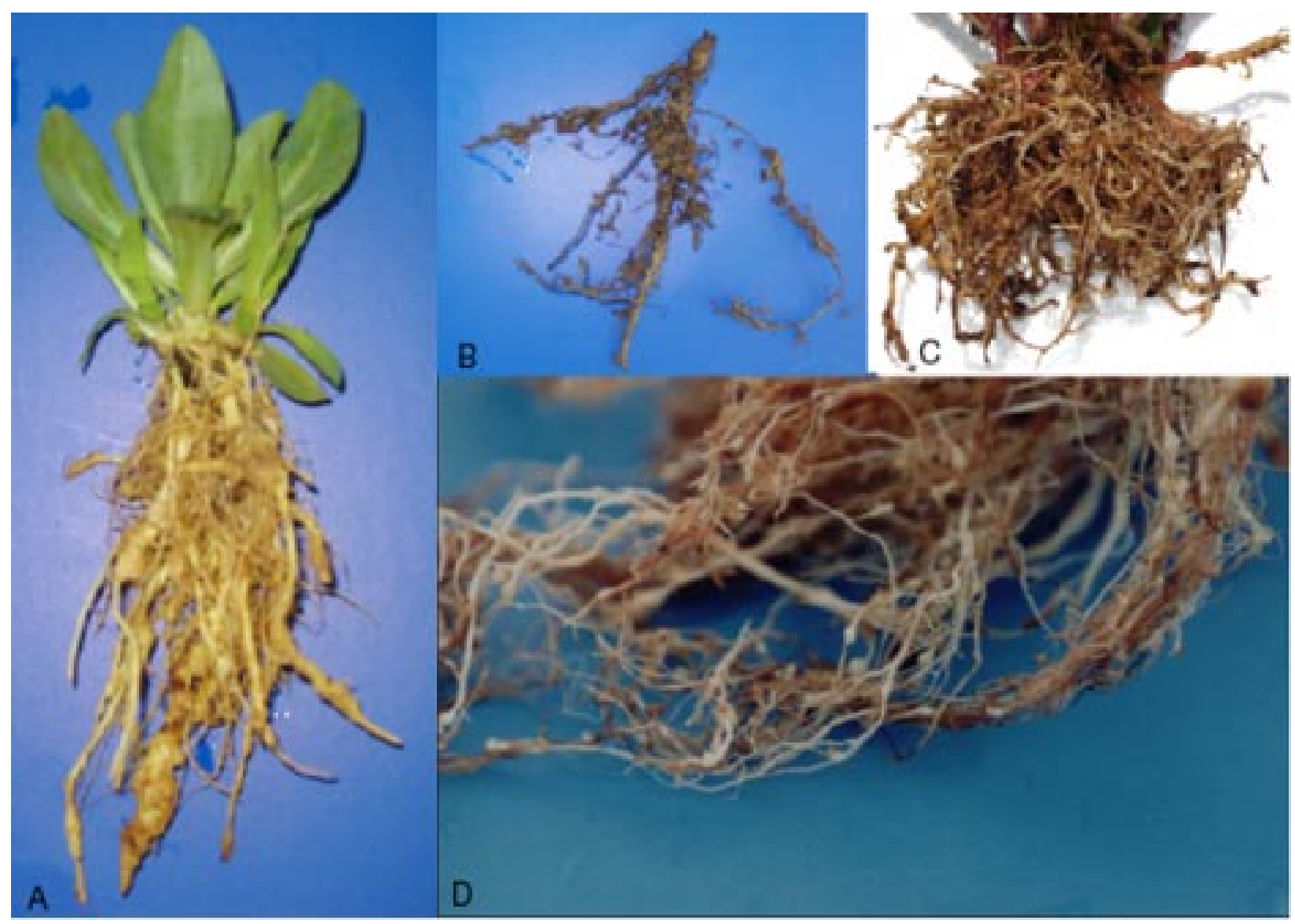

Figura 1. A: galhas radiculares e desenvolvimento insatisfatório de plântula de lisianto infestada por Meloidogyne javanica; B: galhas em roseira causadas por M. hapla; C: galhas em Impatiens balsamina causadas por M. javanica; D: galhas em Exacum affine causadas por M. javanica.

Figure 1. A: root galls and stunted lisianthus seedlings infected by Meloidogyne javanica; B: galls on rose roots caused by M. hapla; C: galls on Impatiens balsamina roots caused by M. javanica; D: galls on Exacum affine caused by M. javanica. 


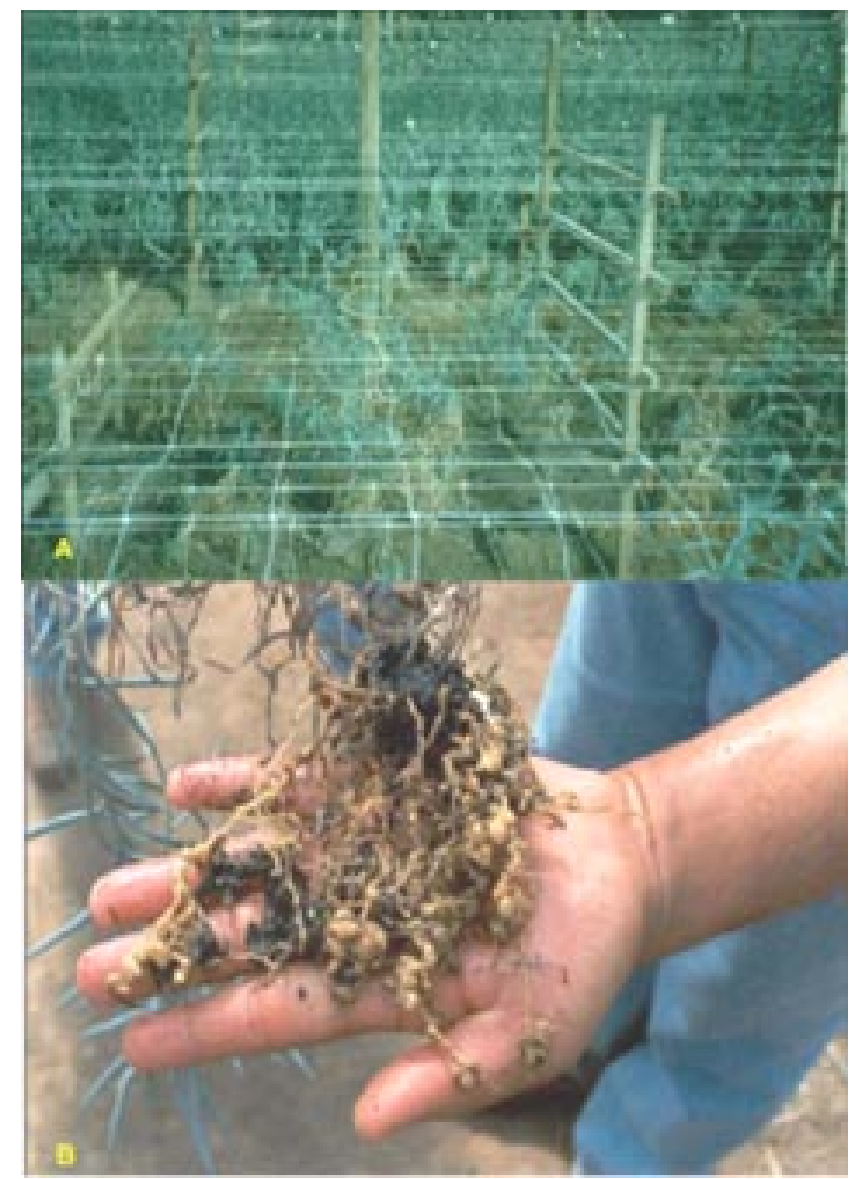

Figura 2. Redução do desenvolvimento (A) e galhas radiculares (B) causadas por Meloidogyne javanica nas plantas de cravo (Dianthus sp.).

Figure 2. Stunted plants (A) and root galls (B) caused by Meloidogyne javanica on carnation (Dianthus sp.)

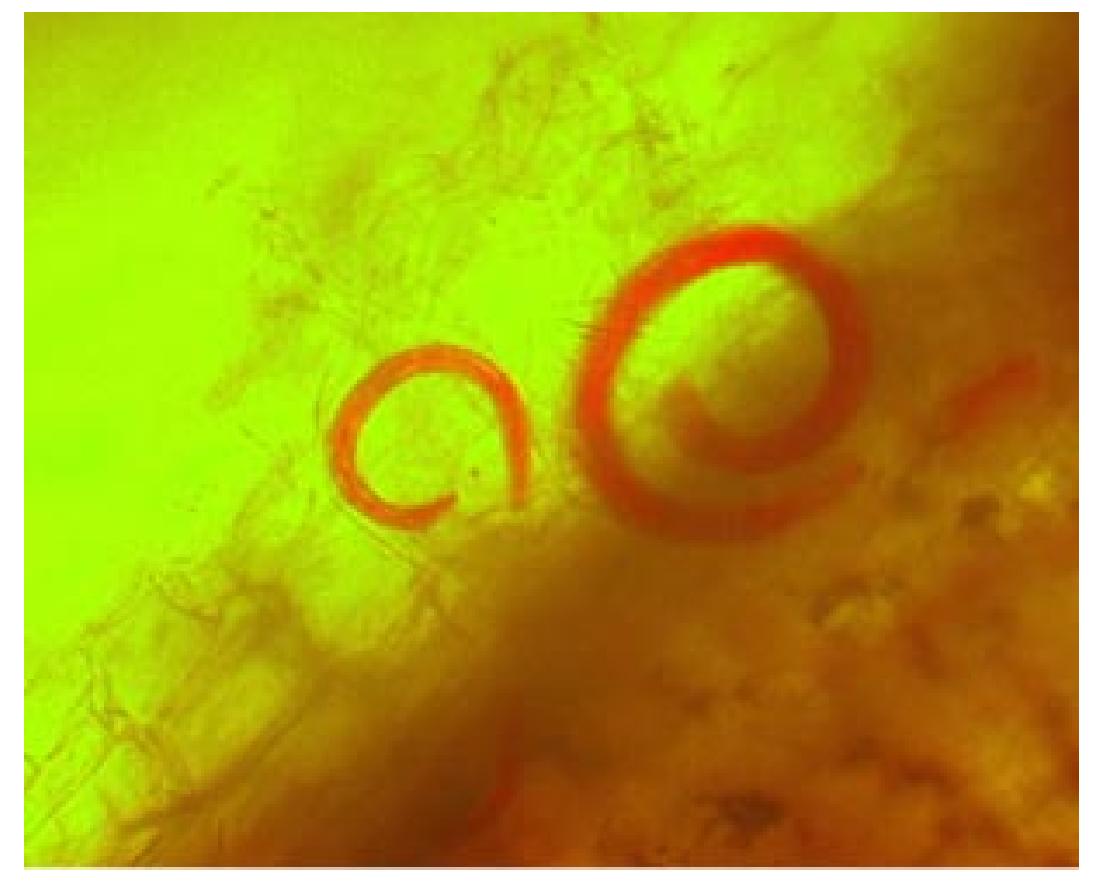

Figura 3. Ectoparasitismo de Helicotylenchus dihystera em raízes de Aptenia cordifolia. Figure 3. Ectoparasitism of Helicotylenchus dihystera on Aptenia cordifolia roots. 
Tabela 1. Nematóides fitoparasitos associados a raízes de plantas ornamentais em municípios dos estados de São Paulo e Minas Gerais

Table 1. Nematodes plants parasitic associated to roots of the ornamental plants in localities of São Paulo and Minas Gerais States, Brazil

\begin{tabular}{|c|c|c|c|}
\hline $\begin{array}{l}\text { Nematóide } \\
\text { Nematode }\end{array}$ & $\begin{array}{l}\text { Planta Ornamental } \\
\text { Ornamental Plant }\end{array}$ & $\begin{array}{l}\text { Tematoides/g de raiz } \\
\text { ematodel } g \text { of root }\end{array}$ & $\begin{array}{l}\text { Procedência das amostras } \\
\text { Origin of the samples }\end{array}$ \\
\hline Meloidogyne arenaria & Cravo (Dianthus cv. Babella) & 606 & Itapetininga, SP \\
\hline \multirow[t]{2}{*}{ Meloidogyne hapla } & Rosa cv. Texas & 1270 & Andradas, MG \\
\hline & Rosa cv. Oseane & 400 & Munhoz, MG \\
\hline \multirow[t]{7}{*}{ M. incognita } & *Gloxinia sp. & 43 & Atibaia, SP \\
\hline & *Orquídea bambu (Arundina graminifolia) & 54 & Campinas, SP \\
\hline & Begonia sp. & 598 & Campinas, SP \\
\hline & Crisântemo cv. Macarrão & 607 & Jaguariuna, SP \\
\hline & Impatiens balsamina & 2110 & Campinas, SP (CEIB) \\
\hline & I. balsamina & 60 & Campinas, SP (Souzas) \\
\hline & *Rosinha do sol (Aptenia cordifolia) & 892 & Campinas, SP \\
\hline \multirow[t]{16}{*}{ M. javanica } & *Hibisco cv. Variegata & 130 & Guarujá, SP \\
\hline & Crisântemo cv. Macarrão & 400 & Jaguariuna, SP \\
\hline & *Hibiscus rosa-sinensis & 84 & Guarujá, SP \\
\hline & *Hibiscus sp. & 2 & Timburi, SP \\
\hline & Camarão amarelo (Pachystachys lutea) & 384 & Guarujá, SP \\
\hline & Cravo (D. caryophilus) & 4080 & Munhoz, MG \\
\hline & *Gloxinia sp. & 40 & Atibaia, SP \\
\hline & *Lisianto (Eustoma grandiflorum cv. King of Snow) & 820 & Ibiúna, SP \\
\hline & *Lisianto (E. grandiflorum) & 6672 & Artur Nogueira, SP \\
\hline & *orquídea bamboo (A. graminifolia) & 54 & Campinas, SP \\
\hline & I. balsamina & 1340 & Campinas, SP (Barão Geraldo) \\
\hline & I. balsamina & 1050 & Campinas, SP (Souzas) \\
\hline & *Falsa ave do paraíso (Heliconia rostrata) & 86 & Piracicaba, SP \\
\hline & *Graptofilo (Graptophyllum pictum) & 2 & Piracicaba, SP \\
\hline & *chapéu-chinês (Holmskioldia sanguinea) & 456 & Piracicaba, SP \\
\hline & *violeta-alemã (Exacum affine) & 600 & Piracicaba, SP \\
\hline \multirow{4}{*}{ Pratylenchus brachyurus } & *Lisianto (E. grandiflorum) & 2 & Várzea Paulista, SP \\
\hline & Hibiscus sp. & 84 & Timburi, SP \\
\hline & *Orquídea (Cattleya sp.) & 14 & Paranapanema, SP \\
\hline & $\begin{array}{l}\text { *Lírio cvs. Sorbone, Tom Pouce, Tiber, Sissi, } \\
\text { Casa Blanca e Time out }\end{array}$ & 6 & Holambra, SP \\
\hline P. zeae & $*$ Rosa & 102 & Holambra, SP \\
\hline \multirow{4}{*}{ Helicotylenchus dihystera } & *Lisianto (E. grandiflorum) & 60 & Várzea Paulista, SP \\
\hline & *Lírio cvs. Sorbone, Tom Pouce e Tiber & 5 & Holambra, SP \\
\hline & *I. balsamina & 15 & Campinas, SP \\
\hline & *Rosinha do sol (A. cordifolia) & 10 & Campinas, SP \\
\hline H. multicinctus & *Camarão amarelo $(P$. lutea $)$ & 24 & Guarujá, SP \\
\hline H. pseudorobustus & *Heliconia sp. & 44 & Registro, SP \\
\hline Cactodera cacti & *Flor de maio (Schlumbergera sp.) & 40 & Jundiaí, SP \\
\hline
\end{tabular}

(*): Novo registro da associação para o Brasil.

New register of the association for Brazil. 